\title{
Assisting Sustainable Forest Management and Forest Policy Planning with the Sim4Tree Decision Support System
}

\section{Floris Dalemans ${ }^{1}$, Paul Jacxsens ${ }^{2}$, Jos Van Orshoven ${ }^{1,2}$, Vincent Kint ${ }^{1,3}$, Pieter Moonen ${ }^{1}$ and Bart Muys ${ }^{1, *}$}

1 Division of Forest, Nature and Landscape, Department of Earth and Environmental Sciences, KU Leuven (University of Leuven), Celestijnenlaan 200E, BE-3001 Leuven, Belgium; E-Mails: floris.dalemans@kuleuven.be (F.D.); jos.vanorshoven@sadl.kuleuven.be (J.V.O.); vincent.kint@ees.kuleuven.be (V.K.); pieter.moonen@ees.kuleuven.be (P.M.)

2 Spatial Applications Division Leuven, Department of Earth and Environmental Sciences, KU Leuven (University of Leuven), Celestijnenlaan 200E, BE-3001 Leuven, Belgium; E-Mail: paul.jacxsens@ees.kuleuven.be

3 Flemish Agency for Nature and Forests, Koning Albert II-laan 20/8, BE-1000 Brussels, Belgium

* Author to whom correspondence should be addressed; E-Mail: bart.muys@kuleuven.be; Tel.: +32-16-329-726; Fax: +32-16-329-760.

Academic Editor: Harald Vacik

Received: 4 February 2015 / Accepted: 12 March 2015 / Published: 24 March 2015

\begin{abstract}
As European forest policy increasingly focuses on multiple ecosystem services and participatory decision making, forest managers and policy planners have a need for integrated, user-friendly, broad spectrum decision support systems (DSS) that address risks and uncertainties, such as climate change, in a robust way and that provide credible advice in a transparent manner, enabling effective stakeholder involvement. The Sim4Tree DSS has been accordingly developed as a user-oriented, modular and multipurpose toolbox. Sim4Tree supports strategic and tactical forestry planning by providing simulations of forest development, ecosystem services potential and economic performance through time, from a regional to a stand scale, under various management and climate regimes. Sim4Tree allows comparing the performance of different scenarios with regard to diverse criteria so as to optimize management choices. This paper explains the concept, characteristics, functionalities, components and use of the current Sim4Tree DSS v2.5, which was parameterized for the region of Flanders, Belgium, but can be flexibly adapted to allow a broader use. When considering the current challenges for forestry DSS, an effort has been made towards the
\end{abstract}


participatory component and towards integration, while the lack of robustness remains Sim4Tree's weakest point. However, its structural flexibility allows many possibilities for future improvement and extension.

Keywords: decision support systems (DSS); forest management and policy planning; ecosystem services; multi-criteria decision analysis (MCDA); Flanders

\section{Introduction}

In recent decades, European forest management has increasingly focused on integrating the multitude of forest ecosystem services within the context of sustainability [1-4]. Forest management planning aims at maintaining a sustainable provision of multiple goods and services, such as timber and non-timber products, carbon sequestration, air and water purification, buffer to natural hazards, climate regulation, biodiversity conservation or recreation [5-7], at the level of the forest management unit [8]. However, the consideration of multiple ecological, economic and social objectives [1] often implies trade-offs and conflicts [9], along with complex interactions on various spatial and temporal scales [10]. The more diverse the demands on forest ecosystem services, the more complex and challenging planning and decision making is $[8,11]$. Simultaneously, sustainable forest management goes hand in hand with a demand for the increased involvement of various stakeholders (land owners, forest managers, land managers, forest industry, forest-dependent communities, policy makers) and the general public in the planning and decision making process [12-14]. This increasing technical and social complexity of forest decision making poses considerable challenges for forest managers and policy makers $[15,16]$, whose choices have a direct and long-term influence on the quality and versatility of the ecosystem services provided by forests.

To accommodate multiple objectives and facilitate participatory decision making in forest management, various decision support systems (DSS) have been developed, mainly in the past three decades [10,17]. These computer-based tools typically combine a simulator, which calculates the outcome of management scenarios using one or several models, with a user interface, a database management system and an optimization algorithm [11]. They may be coupled to a geographic information system (GIS), include visualization technology and allow for stakeholder preferences. By structuring decision problems and analyzing the outcome and implications of alternative management scenarios, DSS greatly facilitate reasoned decision making for stakeholders [10,17]. Available DSS account for a broad range of forest ecosystems [4] and vary widely in terms of functionality (temporal scale, spatial scale, simulated variables, spatial analysis, optimization, visualization), typology (scope and objectives, user involvement, models and methods) and architectural set-up (user interface, database, modules) [18,19]. Comprehensive overviews of available DSS for forest management planning have been compiled within the framework of the European COST Action FP0804 forest management decision support systems (FORSYS); see [4,20].

In the 1980s and 1990s, DSS were addressing relatively narrow, well-defined problems, leading to an abundant collection of specific DSS [10]. These systems were mainly used by scientific researchers themselves for case studies and demonstrations [18]. Later on, driven by the increasing demand for 
integration and participation along with rapid advances in computing hardware and software systems, there was a clear trend towards developing fewer, yet more general-purpose DSS, with a broader scope, more functionalities (e.g., 3D-visualization, optimization and choice algorithms), a modular architecture with linkages to other systems (e.g., GIS tools, web services), larger stakeholder involvement and increasing user-friendliness $[10,11,18]$. This evolution is not at an end, and there still exists a need for further improvement and tailoring of DSS [16]. In this respect, the three following key targets of future DSS development could be synthesized from recent literature. Firstly, there is a need for further integration, referring to the consideration of multiple spatio-temporal scales and a broader array of services [17,21,22], in particular regulating and socio-cultural ones [19]. Several authors recommend a toolbox approach, which refers to a modular software architecture flexibly combining different models and systems $[8,23,24]$ and enabling linkages with different (web-based) data sources and/or other DSS [24-26]. With regard to the current lack of integration, the fact that most DSS are case-specifically developed and are not systematically recorded in a meta-database of DSS [20] is leading to inefficiencies and overlap in development efforts [19]. Secondly, there is a need for more robust outputs, which account for the risks and uncertainties in forest management and policy, related to the increasing exposure of European forests to climate change and natural disturbances (heat waves, drought, fire, storm, flooding, biotic risks) [27-29] or to the future development of the wood market and other human needs. In this context, there is a need to generate outputs that consider factors of resilience, such as the genetic variation between provenances [30,31] and the occurrence of mixed and uneven-aged stands within the forest [11]. Lastly, the success of approaches for participatory decision making has been limited so far $[13,15]$. Although multiple obstacles for successful stakeholder involvement might exist in the entire planning cycle [14], with regard to DSS design, emphasis should be put on the inclusion of tools that help with assessing options, such as multi-criteria analyses, voting models, cognitive mapping, visualization techniques and web-based tools [13,26], as well as on the customization of the DSS (interface, support) towards the specific type(s) of users, in order to obtain user-friendliness, transparency, credibility and, ultimately, effective adoption [13,15,32]. The identification of the users and their needs, as well as user involvement in DSS development and testing are crucial with regard to the latter [13].

In addition to these three key targets, an elaborate and more practically-oriented set of empirical guidelines for the development and implementation of DSS has been synthesized by [16] from past experiences and lessons-learned of the experts involved in the FORSYS action. Similarly, a lot of these are obvious, yet lacking conditions for generalized use of DSS [11].

Future prospects indicate a continued increase in the demand for integration and participation, in the labor costs for inventories and field observations and in computer power. Therefore, the development of multifunctional, reliable and user-friendly DSS will become an even more essential and economically relevant challenge for flexible and interactive forest management and forest policy planning $[11,19]$. The current shortcomings, as well as newly emerging decision problem domains propel the demand for new DSS from audiences ranging from individual forest managers to landscape planners to (supra-)national policy makers and administrations [9,10,17].

This paper presents the Sim4Tree DSS, which has been developed taking into account several of these issues. The concept and characteristics of Sim4Tree, its current functionalities and its software components will be elucidated in Section 2. Section 3 will illustrate Sim4Tree's practical use and applicability by means of a case study for Flanders, Belgium, the region where it was developed. The 
strengths, weaknesses and opportunities of Sim4Tree will be evaluated against the three aforementioned key targets in Section 4.

\section{The Sim4Tree Decision Support System}

\subsection{Concept and Characteristics}

Sim4Tree supports strategic (long term) and tactical (medium term) forestry planning by providing simulations of forest development, ecosystem services potential and economic performance through time, from a stand to a regional scale, under various management and climate regimes. Sim4Tree allows comparing the performance of different scenarios with regard to diverse objectives so as to optimize the management choices. Its main characteristics are seven-fold.

\subsubsection{Decision Support Based on Best Available Models and Data}

Sim4Tree is not a model or simulator in its own right, but a decision support tool in which modelling outputs from very different models or simulators (e.g., yield tables, mechanistic ecosystem models) can be flexibly plugged in and connected to an intuitive user interface and management optimization routines. Models are not coupled to the DSS in real time; rather, model runs are taken in a tabular format following a predefined structure. The actual Version 2.5 is designed for use in Flanders, using best available forest data and models for the region, but the flexible database structure of Sim4Tree and the open source design allow for an easy adaptation for use in other geographical locations with a different decision environment.

\subsubsection{Three Decision Levels: N1 to N3}

Sim4Tree is conceived as a toolbox, rather than as one overdesigned, all-round tool, which would have difficulty matching the needs of all users. This is reflected in separate decision levels for targeted use at three spatio-temporal scales. The first level (N1) (The letter "N" in each level designation refers to the Dutch equivalent for level, i.e., "Niveau") can tackle strategic policy questions at a regional extent, the second level (N2) strategic management questions at the forest management unit level and the third level (N3) tactical management questions at the stand level. In the current v2.5, only N1 and N2 are operational, while for N3, the conceptual and technical basis is provided.

\subsubsection{Spatial Characterization of the Forest by a Set of Pixels}

At all three decision levels, the forest area is represented by a grid of independent square spatial units (pixels) and correspondingly stored in the database. Each pixel is characterized by one soil type, one tree species category (or other forest-related land use type, e.g., forest gap, heathland) and one age class, and no within-pixel variation is considered. Forest development comes down to each individual pixel delivering ecosystem services and producing certain costs and benefits through time. The spatial resolution of the pixel grid varies for the three decision levels, in line with the spatial character of the corresponding problem type: pixels are $10,000 \mathrm{~m}^{2}$ in $\mathrm{N} 1,1000 \mathrm{~m}^{2}$ in $\mathrm{N} 2$ and $100 \mathrm{~m}^{2}$ in $\mathrm{N} 3$. The time 
resolution equals five years in $\mathrm{N} 1$ and $\mathrm{N} 2$ and one year in $\mathrm{N} 3$, while forest development can be simulated for 150 years in N1, 90 years in N2 and 20 years in N3.

As a consequence of these conceptual choices, Sim4Tree does not account for interactions between pixels. For example, although mixed and/or uneven-aged stands can be initialized with an appropriate pixel set, no effects of species or age interactions will be simulated. Furthermore, Sim4Tree is not suitable for simulation of ecosystem services that cannot be easily attributed to individual pixels (e.g., recreational value is often attributed to a larger extent) or that require neighborhood analyses between pixels (e.g., air pollution filtering depending on canopy surface roughness).

\subsubsection{Forest Management Scenarios}

Forest development is directly driven by a user-defined management scenario, summarizing choices on afforestation and deforestation, regeneration area and management of current and future generation stands and habitats. Species-specific management choices essentially comprise the silvicultural regime, the timing of final cut and regeneration options. Choices on silvicultural regime are directly determined by the extensiveness and flexibility of the available growth models. All management choices can be differentiated based on geographic location, site suitability, owner category and/or legal status. The defined management choices are correspondingly assigned to the individual pixels.

\subsubsection{Climate Change Scenarios}

For each simulation of forest development, a climate change scenario has to be selected. In Sim4Tree $\mathrm{v} 2.5$, these are either a baseline scenario (no climate change), a weak climate change scenario or a strong climate change scenario. The latter two correspond to the two extreme scenarios in the Special Report on Emissions Scenarios (SRES) report by the Intergovernmental Panel on Climate Change (IPCC), respectively the B1- and the A1F1-scenario [33]. Depending on the used model type, Sim4Tree can either directly use model output (restricted to mechanistic models that allow simulation of climate change) or assume a climate-driven change in site suitability (e.g., for climate-invariant empirical yield tables).

\subsubsection{Forest Development}

At the start of the simulation, each individual pixel is assigned a tree species and age class (or forest-related land use type), a corresponding site suitability and a silvicultural regime. Site suitability is derived from soil type and prevalent climate in terms of yield potential (volumetric increment) and discretized into five ordinal yield classes for each species. User-defined management choices are translated into changes of the pixel composition and attributes throughout time. Individual pixel development is then correspondingly derived from the user-chosen model, determining its ecosystem services provision and cost-benefit structure at each time step. Forest development is derived by integration of individual pixel development. The whole procedure is implemented in an open source relational database management system (RDBMS) through SQL statements. 


\subsubsection{Types of Questions}

Sim4Tree can be used to solve different types of questions [34]. The first type of question ("what if") has the general structure "What is the outcome, if certain parameters are chosen?", where the outcome refers to ecosystem services and/or realized costs and benefits, while the parameters refer to all user-defined options. In the context of decision making, however, managers and policy makers usually aim at optimizing management choices. This concerns other question types, such as the "how" question ("What should management be like to obtain an optimal outcome?"), the "where" question ("where to apply a certain management to obtain an optimal outcome"), the "how long" question ("how long to apply a certain management to obtain an optimal outcome") or a combination of these. These question types require an optimization module.

\subsection{Sim4Tree v2.5 Functionality and Flexibility}

Sim4Tree v2.5 (January, 2015) is designed for and implemented for use in Flanders (northern Belgium). Nevertheless, most functionality is extendable to other regions or site conditions, given appropriate adaptation of the software. In this section, the current functionality is described, as well as how it can be improved and extended.

\subsubsection{Initialization}

Because of the large spatial extent, on decision level N1, the forest composition at time step zero (reference year 2010) is pre-initialized and cannot be altered. Conversely, on levels N2 and N3, the initialization should be provided by the user. In both cases, it is possible to subdivide the area into different geographic units. On N1, various options are included for Flanders: e.g., provinces, ecoregions, management regions, special protection/conservation areas. Areas on N2 and N3 can be subdivided into zones and stands. Similarly, the pixels can be assigned a legal status and an owner category. The geographic units, legal status and owner categories are equally pre-initialized and unchangeable on N1, while the user should define them on levels N2 and N3. They enable two important functionalities. Firstly, simulations can be run for only one or several units (or statuses or categories) instead of for the entire initialized area, which might be useful for specific studies wherein only a specific unit is of interest. Secondly, even when the simulation is done for the entire initialized area, the subdivision allows management to be separately defined for and differentiated between the constituting units (or statuses or categories).

The initialization includes the characterization of the pixels by one soil type, using the Belgian soil classification system, by one tree species category (or other forest-related land use type) and one age class. Sim4Tree v2.5 is mainly operational for high forests, and while other forest-related land use types (coppice, forest edges, forest gaps, heathland, water and other non-forest areas) can be assigned to a pixel, their development and corresponding ecosystem services provision cannot be simulated in Sim4Tree v2.5, due to a lack of appropriate models for Flanders. Correspondingly, these pixels are only accounted for by the area statistics.

The software easily allows other regions and countries to be added on N1. This is conditional on the availability of $10,000 \mathrm{~m}^{2}$-scale forest maps containing the necessary information on land use, species, 
age class and soil type. Effective implementation might also require minor changes to the graphical user interface (GUI), if geographic subdivisions, legal statuses or owner categories do not match those for Flanders. Analogously, the development of land use types other than high forest could be simulated, given the availability of appropriate models.

\subsubsection{Model Choice}

Pixel development through time is derived from best available models for Flanders, which are stored in the database in tabular format following a predefined structure. Models are of crucial importance, since they determine which tree species and ages, land use types, silvicultural regimes and ecosystem services can be accommodated. In Sim4Tree v2.5, a set of empirical growth models is used, based on Dutch [35] and Flemish [36] yield tables. These describe the evolution of even-aged, monospecific stands with optimal tree density, in terms of stand and harvest dimensions, with a temporal resolution of five years. Data are available for all common species of the region, while less common species are set equal to a common species based on expert knowledge (see Supplementary Materials, Table S1). These yield tables have been extrapolated towards higher age categories and reclassified into five yield classes, linked to five site suitability levels. The main drawbacks of this empirical model are the limited set of silvicultural regimes for high forest that it allows, the limited set of ecosystem services that it predicts and its inability to model the development of stands with suboptimal tree density and of other land use types.

A special note should be added on the specification of the climate change effect in the model. Since empirical models, such as yield tables, predict growth assuming a fixed site suitability, they cannot be used for the climate change scenarios. Still, to account for climate change using these models, a hybrid approach has been adopted [11]. While in the baseline scenario (no climate change), site suitability is considered invariable for a pixel, the climate change scenarios might imply changes in time of site suitability for a pixel. These changes are based on estimated species-specific climatic responses, species ecology, expert knowledge and climatological projections for Flanders. More specifically, for each combination of species and soil type, a corresponding discrete site suitability has been modeled as a step function of time. At each new generation, this function determines whether a shift of site suitability/yield class should occur for a given species, as well as the magnitude and direction of this shift. Obviously, this approach comprises certain shortcomings. Firstly, it is an artificial and discretized estimation of the ecophysiological and multi-directional effect of climate change. Secondly, it only includes direct temperature and precipitation effects, while not accounting for other effects, such as atmospheric $\mathrm{CO}_{2}$-enrichment and natural hazards. Thirdly, since the SRES-scenarios do not serve as a direct model input, the accuracy of the estimation is directly dependent on the expert interpretation. These shortcomings would largely be resolved by coupling a mechanistic (e.g., ecophysiological) model into Sim4Tree, which can account for a varying climate in modeling growth paths.

In general, while the current models for Flanders imply certain limitations, a major part of the flexibility of Sim4Tree is owed to the fact that it has not been grafted on this or any other particular model and that its design enables one to accommodate a wide variety of models. These can, for instance, account for other regions, species, land use types, management options, climate changes scenarios and ecosystem services, as long as they can be added in a pre-calculated tabulated format. The procedure of 
plugging-in models is however not yet automated and may still require minor adaptations to the software according to the specific features, requirements and outcomes of the model.

\subsubsection{Forest Management Options}

To influence the overall forest structure and composition, the user can define targeted land use changes for pixels, as well as introduce 'new pixels' (which were not assigned a species or forest-related land use types during the initialization) to enable afforestation and nature expansion. With regard to specific land use management, options are only available for high forests in Sim4Tree v2.5. The user can first designate for each species a fraction of the high forest as non-productive, for which, analogously to the other land uses types, development is not simulated. For the remaining productive high forest, species-specific options for the silvicultural regime, timing of final cut and regeneration are available:

- The availability of choices for silvicultural regime depends on the model used. In Sim4Tree v2.5, based on yield models for Flanders, the choice is limited to planting density (e.g., poplar) or thinning intensity (e.g., Corsican pine).

- The time of final cut it is either fixed at a certain age or target diameter or determined by gradual conversion with settings for the conversion period and the age range where conversion should be avoided.

- Regeneration is defined by species choice. Users may either select one species or define a set of species. In the latter case, a probabilistic algorithm distributes the species over the pixels according to an assigned priority and in such a way that species can be put either on the most suitable locations or that overall productivity is maximized.

- Sim4Tree simulates final cut as a clearcut and regeneration as planting of individual pixels. However, by appropriate pixel selection and timing, also group selection or shelterwood cuttings with gradual regeneration can be simulated, especially at N2.

Finally, the overall annual area of final cuts can be limited to a user-defined maximal value, for reasons of feasibility of workload or to meet sustainability standards. If this limit is exceeded in a certain time step, the clearcut in excess is automatically postponed, according to an algorithm that assigns a priority to each pixel. As such, all management rules are ultimately executed, but their initial timing may be overruled. The software assists the user in setting this upper limit by providing a measure of the mean expected clearcut area assuming a normal forest.

\subsubsection{Cost-Benefit Options}

Besides simulations of forest composition and ecosystem services, Sim4Tree can also provide an economic analysis of management scenarios. It allows one to take various costs and benefits into account: the software includes pre-defined settings for species-specific management costs (e.g., planting, pruning, tending) and benefits (timber prices, differentiated by diameter class), yearly costs (e.g., overhead, transaction costs), subsidies (specifically for Flanders) and various other benefits (yearly or at a specific moment in time). The user can decide to either exclude or add each of these to the simulation, modify their values and timing and add additional costs and benefits, which are not included by default. 
To account for the temporal dimension of costs and benefits, it is possible to define a rate of return for discounting towards the net present value (NPV).

In terms of database functioning, the costs and benefits are calculated for each time step and for each pixel according to its assigned silvicultural regime and development. If new models would allow for additional silvicultural regimes, the corresponding cost and benefit items can be added to the economic module.

\subsubsection{Output}

Composition, ecosystem services and cost-benefit structures are calculated for each pixel and for each time step in a tabular output. This full detailed output can be exported from the software for subsequent analysis or visualization in external software. Within Sim4Tree itself, the results can be aggregated and/or detailed both spatially (per species, geographic unit, legal status and/or owner category) and temporally (per time step or for the entire simulation period) and can be easily visualized in tables, graphs and maps (see Supplementary Materials, Figure S1).

Sim4Tree provides standardized output on forest composition and structure (e.g., area, age structure), diversity (e.g., species diversity, fraction of native/hardwood species, age diversity) and costs-benefits throughout time. The ecosystem services that can be calculated, are highly dependent on the model that is used, more specifically on the nature, temporal resolution and extensiveness of the development path provided by the model. Based on yield tables, Sim4Tree v2.5 derives output on timber production (e.g., increment, roundwood yield, regeneration) and, via expansion and conversion factors, on biomass yield and $\mathrm{C}$-sequestration in trees.

The database structure is designed to allow the addition of extra variables to the DSS output by programming the appropriate SQL statements. In this way, also other ecosystem services for high forests or for other land use types (e.g., related to soil C, water, timber quality) can be added, conditional on the availability of appropriate source data for the models that are added to the software.

\subsubsection{Optimization Options}

Sim4Tree v2.5 is able to answer the "what if" and the "how" questions (see earlier). To answer the "what if" question, the user should solely interpret the Sim4Tree output of the simulated scenario based on the particular parameters. In addition, an optimization module is included for the "how" question. It enables users to compare the outcome of different management scenarios for a forest area, based on identical model, climate and simulation time choices. Importantly, this optimization is relative rather than absolute, meaning that Sim4Tree can only compare predefined scenarios, while it is not able to identify the most optimal approach out of every possible set of applicable management options [37]. Optimization is achieved by means of a multi-criteria decision analysis (MCDA) approach [38]: scenarios are compared based on their values for certain ecosystem services and/or economic variables (= multi-criteria), either at a given point in time or cumulatively over the simulation period, which allows stakeholder preferences to be included in terms of the criteria that are selected and their weights. In addition, the user can opt to exclude certain scenarios from the optimization by defining threshold values for ecosystem services provision and/or economic performance that should be met. Non-excluded scenarios are ranked by means of the iterative ideal point thresholding (IIPT) algorithm [39,40], after 
which the optimal scenario(s) can be identified. Note that the optimization module can be analogously used to investigate the sensitivity of a management approach to the cost-benefit parameters; in this case, these parameters are varied instead of the management options, and optimization occurs only with regard to economic performance.

\subsubsection{Language Choice}

In accordance with the current application of the DSS in Flanders, Sim4Tree v2.5 and all of its documentation are available in Dutch. Translation to other languages is straightforward.

\subsection{The Sim4Tree Components}

As a DSS, Sim4Tree flexibly integrates various components in a logical workflow (see Figure 1). Several of the features in this workflow have already been discussed; this section will describe the remaining ones.

\subsubsection{Database Management System}

The database management system links the models, initialization, user choices, output and optimization components with each other in a modular structure (see Supplementary Materials, Figure S2). It is implemented in the free software package, PostgreSQL. In practice, a client-server model is being used, where clients locally install a software and a database component, which are automatically updated according to the central database, which is hosted and managed on a server. Sim4Tree is only developed for use with the Microsoft Windows (XP Service Pack 3 or higher) operating system.

\subsubsection{Graphical User Interface}

The graphical user interface (GUI) adds functionality for the users, by guiding them in a step-by-step approach through the different DSS settings (initialization, climate and management scenarios, cost-benefit parameters and optimization) and by displaying the output (see Supplementary Materials, Figure S3), while translating correspondingly to and from the database via queries throughout. The GUI has been developed in an attempt to optimally combine intuition and performance, with strong feedback from and interaction with the users. It is a stand-alone application that has been developed using the free software framework, C\#.NET 4.

\subsubsection{User Manual and Support}

The GUI provides access to three user support materials. Firstly, the extensive on-line user manual explains the concept and all functionalities of Sim4Tree and provides examples, useful links and background information. Secondly, the Sim4Tree final report contains the contextual and technical information. Lastly, a feedback form is provided in which specific questions or remarks to the software administrator can be filed. All of these are hosted and managed on a server, but are currently only available in Dutch. In addition, interactive training sessions, including follow-up, have been designed and organized for a variety of stakeholders and potential users. 
(a)

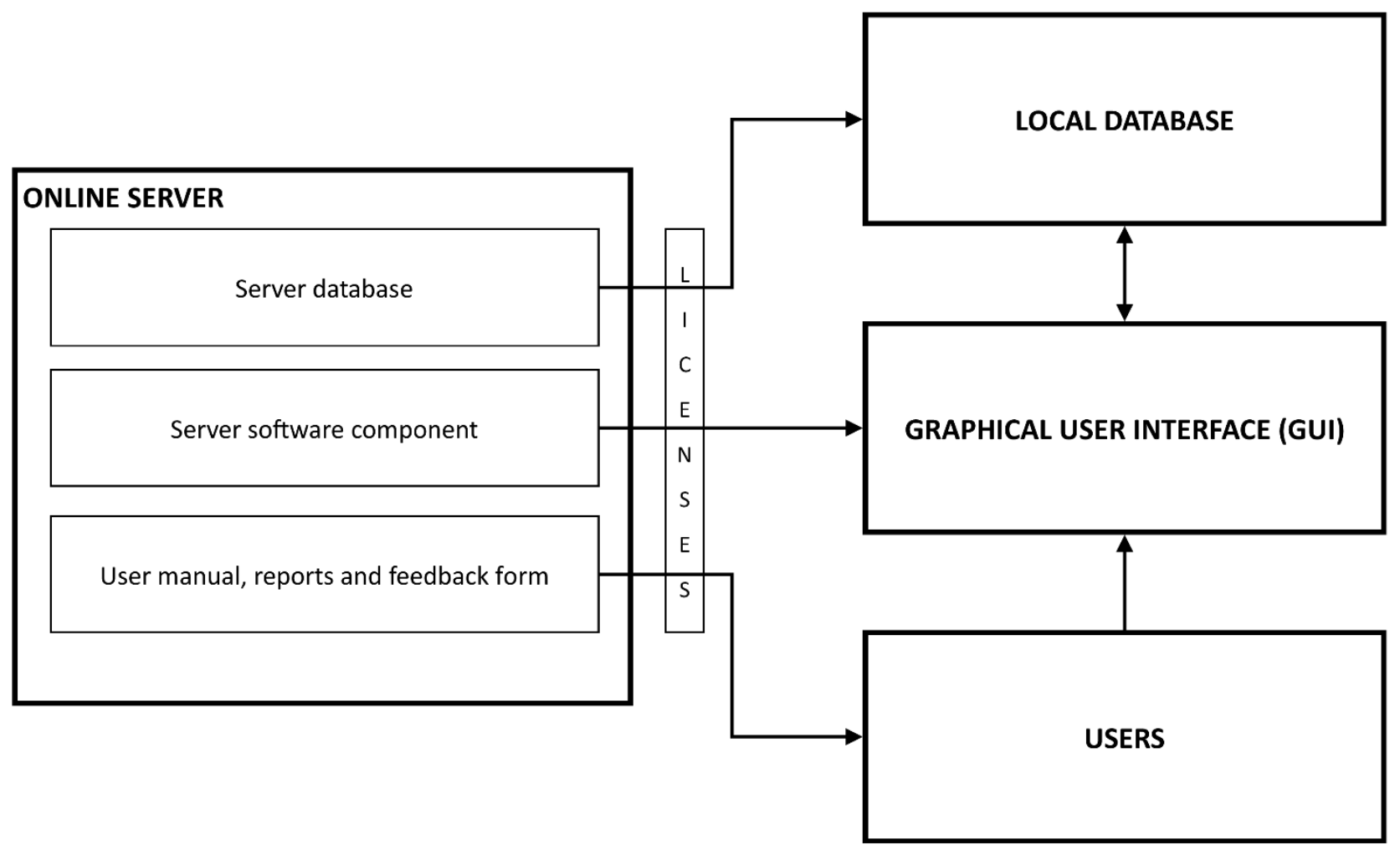

(b)

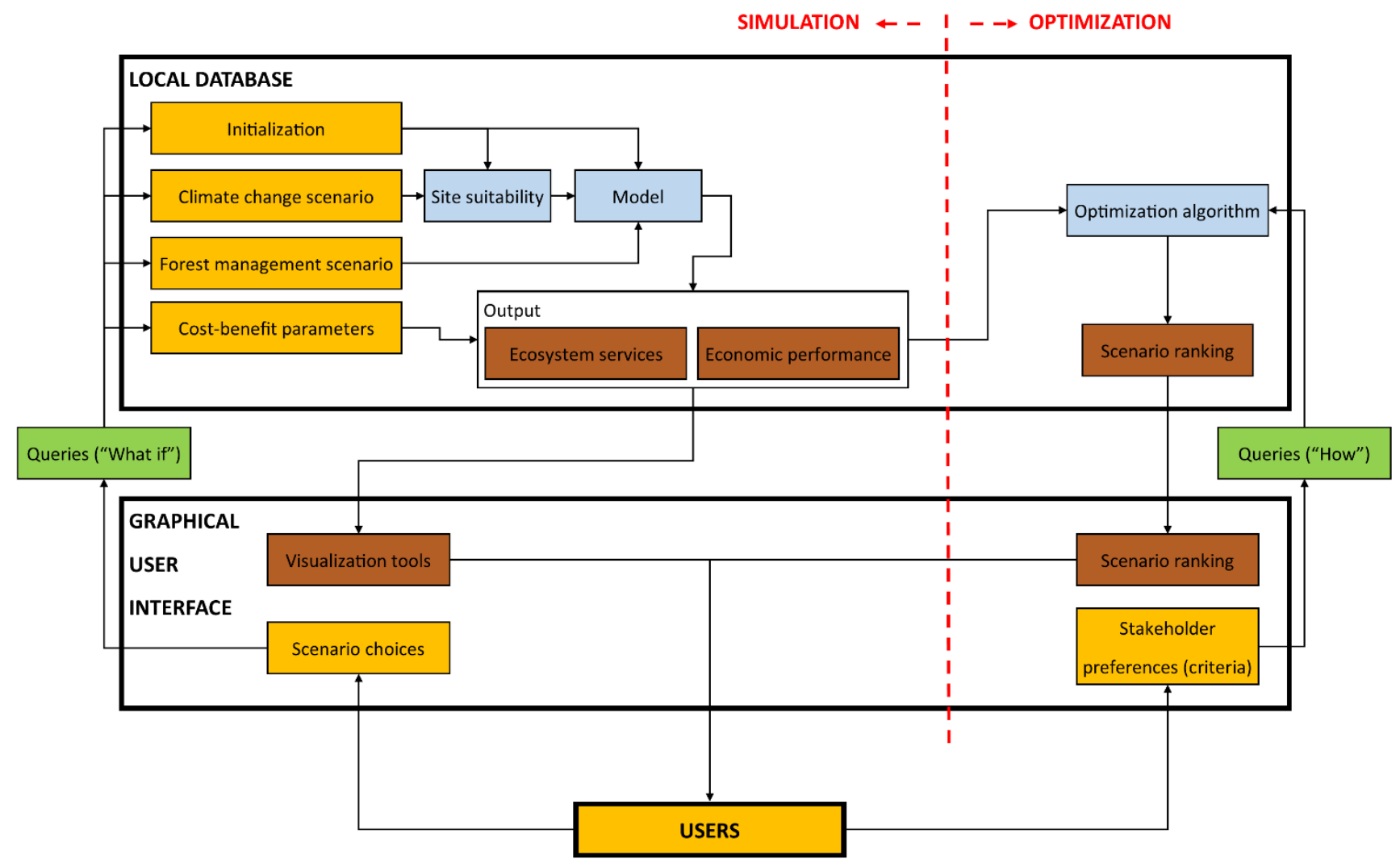

Figure 1. Sim4Tree components and workflow. (a) Communication between the server and the local components; (b) workflow of Sim4Tree. 


\subsubsection{Licenses}

Sim4Tree combines different existing datasets and tools and adds functionality within a DSS. The licenses and restrictions for use of these datasets and tools apply when using the software. The DSS itself is owned by the Flemish government, which has the exclusive right to issue licenses for third party use. Sim4Tree v2.5 is licensed free of charge for all interested users.

\section{Case Study: The Use of Sim4Tree for Participative Decision Making on the Strategic Management of the Forest Complex "Bosland"}

Sim4Tree's potential for supporting multi-stakeholder strategic decision making is illustrated by its use in the Bosland project. The Bosland project aims at sustainable forest management in the $\pm 45-\mathrm{km}^{2}$ forested area of Bosland, situated in the northeast of Flanders, to provide a multitude of functions (e.g., timber production, conservation area, recreation) for a variety of stakeholders (e.g., owners, forest industry, visitors). This vision translates into the management plans for the diverse owners. These drafted individual plans, however, consist largely of ad hoc management interventions and do not provide an assessment of the future overall forest development. As such, the Bosland association felt the need for an overarching strategic management plan. This is where Sim4Tree came in: based on the state of the forest in 2010, the effect of different management scenarios on the forest development up to 2070 was simulated, and the optimal scenario was identified in a participatory process.

\subsection{The Bosland Forest and Management Objective}

The Bosland project area consists of about $93 \%$ of forested area, where the remaining $7 \%$ is made up of small patches of non-forest habitat, water bodies and infrastructure. The forest largely consists of homogeneous stands on sandy soils and is dominated by Corsican pine (Pinus nigra var. maritima) and Scots pine (Pinus sylvestris), which constitute $94 \%$ of the standing stock. The envisaged management for the forest complex essentially comes down to a targeted conversion of stands to achieve a more heterogeneous forest in terms of tree species, tree ages and habitats, while maintaining reasonable harvests and growing stock. More specifically, this implies striving for a larger fraction of native hardwood species, high-quality species and trees, non-productive forest and other habitats, while allocating management types and conversions at the most optimal places, at the most optimal time. Since the creation of additional non-productive forest and other habitats, which is tightly linked to recreational and conservation functions, will occur in predefined zones, the objective for Sim4Tree was to support the strategic planning of conversions towards native hardwood species and high-quality species and trees.

\subsection{Simulation and Optimization of Management in Sim4Tree}

The defined objective can be achieved in various ways. To structure the plethora of choices, a hierarchy containing three essential management options, each including sub-options, was designed and used to define 36 management scenarios (see Figure 2). These were correspondingly differentiated in Sim4Tree, giving rise to 36 scenarios, which were then simulated (to illustrate this, the essential scenario parameters are listed in the Supplementary Materials, Table S2). To illustrate the influence of the various 
management options, the evolution in species composition is displayed for three scenarios in the Supplementary Materials, Figure S4.

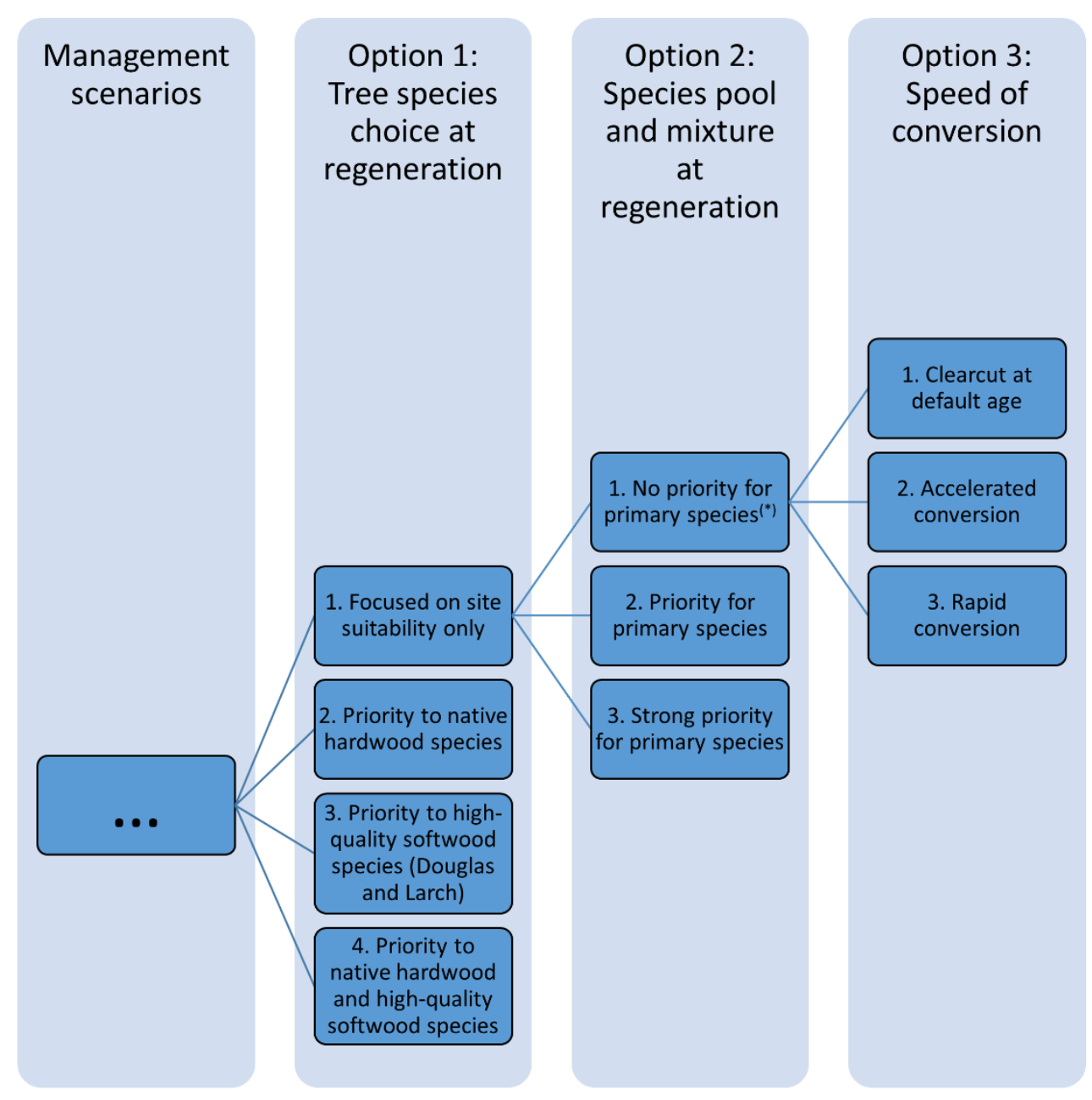

Figure 2. Overview of the hierarchical structure in management options for Bosland. Management scenarios are defined by choosing amongst three hierarchical management options. The name of a scenario (e.g., 312) indicates the chosen sub-options. By considering all possible combinations of sub-options, a total of 36 scenarios is defined. * The differentiation between primary and secondary species depends on the tree species choice at regeneration and the site quality.

Subsequently, the optimal management scenario was identified in four steps. In the first step, each scenario was held against a set of ecosystem service threshold values and excluded in case of non-compliance. More specifically, it was based on compliance of the forest state in 2070 with the current forest legislation, which consists of a set of norms and principles for sustainable forest management composed by the Flemish government. In a second step, the remaining scenarios were compared based on their provision of 14 different ecosystem services (see Supplementary Materials, Table S3), which reflect the targeted management objectives and, by extension, the Bosland project aims. The scenarios were ranked using the IIPT algorithm (see Supplementary Materials, Table S3), using a set of criteria weights defined by the stakeholders (see the next section). In a third step, the data were exported and subjected to an alternative MCDA, using the program, Web-Hipre [41] (see 
Supplementary Materials, Figure S5), as well as to a sensitivity analysis to account for the effects of weight choices, using the program, JSMAA [42] (see Supplementary Materials, Table S4). In the last step, the three highest ranked scenarios were selected and presented to the stakeholders (see the next section).

\subsection{The Participatory Process}

Although Sim4Tree was vital for providing the technical information, based on which the strategic management plan could be designed, the effective realization of this plan was a result of a broader participatory process. Indeed, Sim4Tree both required input from a stakeholder committee, which included representatives from the forest managers, the municipalities, the wood trading and processing industry and local interest groups, and, in turn, equally only provided input for the eventual decision making. With regard to input for Sim4Tree, the development of the specific set of management scenarios for attaining the envisaged objective, i.e., the definition of the different scenarios, was only possible thanks to the stakeholders' expertise and was based on differentiating expectations with regard to future wood market, policy development and climate change. Likewise, the criteria and weights for evaluating the different management scenarios against the envisaged objective were decided upon by the committee. In turn, Sim4Tree offered the input for the additional MCDA and the sensitivity analysis, which all together offered the stakeholders a scientifically-founded set of optimal scenarios, amongst which the most optimal one, however, was eventually chosen not solely based on its quantified rank, but on a holistic and expertise-guided assessment of its performance. In addition, the committee provided specific suggestions for further refinement and optimization of the three selected management scenarios, whereafter the simulation-optimization-selection cycle could be repeated. Finally, the most optimal scenario only formed a blueprint, subject to the limitations of the software, based on which the stakeholders drafted the final strategic management plan, which was then translated into the individual management plans.

\subsection{Shortcomings: Accuracy and Uncertainty in Sim4Tree}

By using mathematical descriptions and concepts to describe the real-world forest development, Sim4Tree's simulations inevitably imply inaccuracies. Conceptually, we can distinguish between three sources of inaccuracy. Firstly, the database concept requires pixels to be independent, implying that neither interactions nor explicit spatial ecosystem services can be simulated (see earlier). Secondly, there are inaccuracies related to the input data. This comprises, on the one hand, measurement errors of the initial forest status (initialization). For example, for Flanders as a whole, the initialization (N1) is estimated to have a maximum deviation of $10 \%$ with regard to forested area and a deviation of respectively up to $5 \%$ and $20 \%$ for the area of common and uncommon tree species, due to lack of a single, up-to-date map for the distribution of tree species in Flanders. On the other hand, this refers to the uncertainties for the future biotic and abiotic environment, such as climate change (the SRES-scenarios) and the future wood market (cost-benefit structures). Lastly, the model introduces inaccuracies by not or only partly accounting for the effect of certain factors. The yield tables can, for instance, only account for a very specific subset of contemporary management regimes and for time 
steps of five years, while they cannot account at all for factors such as genetic effects and pest and disease dynamics.

The three sources of inaccuracy add to each other. To assess overall inaccuracy, simulated and measured standing stock were compared for short-term simulations of five forests on level N2. Differences between $1 \%$ and $20 \%$ were typically observed, with outliers up to $80 \%$ for individual species. Nevertheless, this should be put into perspective for three reasons. Firstly, when enlarging the area (N1), these deviations are expected to largely eliminate each other. Secondly, when different scenarios are compared to each other for optimization, the scenario errors are expected to be very similar and, therefore, will only marginally influence the outcome of interest. In a general sense, Sim4Tree does not claim to make exact predictions, rather the results should be interpreted in relative terms by comparing scenarios. Lastly, and most importantly, only the inaccuracies due to the database concept are inherent to the Sim4Tree DSS, while the others stem from the "external" data and tools that it uses. It has shown that the software can flexibly replace and/or integrate these with new improved data and tools (see earlier).

\section{Discussion}

Although Sim4Tree has been developed in first instance as a result of the lack of instruments assisting adaptive forest management planning tailored to Flanders, along the way, needs were revealed corresponding to the key targets of DSS development (see the Introduction). Indeed, the forestry sector in Flanders is equally confronted with the complex challenges of sustainable forest management in a dynamic environment, and as such, the inclusion of multiple spatio-temporal scales and ecosystem services, the demand for robust results and the possibility of participatory decision making were pertinent to the design of Sim4Tree all the way through. Accordingly, the software can be considered an effort towards addressing these three key targets of forest management DSS development and was held against these.

With regard to integration, Sim4Tree is an example of how a toolbox approach can be used to support multiple spatio-temporal scales. The pixel concept can be applied to any spatial scale, while the required detail, in terms of temporal resolution and management options, can be correspondingly adjusted by adding the output of a suitable model to the database. The plug-in architecture of the database allows for this and the integration of other data sources (e.g., other geographical datasets) and methods (e.g., other optimization algorithms), although the model flexibility does not extend to real-time models. In this respect, Sim4Tree could be considered a DSS generator, while Sim4Tree v2.5 is then a specific DSS [43]. The ease of plugging-in equally accounts for the GUI, which can be extended with additional components, such as other GIS plug-ins, visualization tools and ecosystem services. This might be mostly necessary for the latter: the currently limited array of ecosystem services, the predominant focus on timber production and the impossibility of including explicit spatial ecosystem services remain main shortcomings. Nevertheless, it was shown in the Bosland case study that this can be solved by calling in Sim4Tree for only a part of the considered ecosystem services, which makes it a part of a bigger toolbox. In this respect, the software cannot yet be automatically linked to other DSS or services, yet its adherence to the Microsoft Windows operating system and PostgreSQL RDBMS implies favorable conditions. From another viewpoint of integration, it should be noted that the fundamental design of Sim4Tree is 
not geographically specific and that it has been added to the ForestDSS meta-database [20] to promote visibility and cooperation.

The lack of robustness of the DSS is for now the weakest point. Yield tables for Flanders are only available for a very limited set of management regimes and should only be considered as an approximation in light of the recent innovative and diversifying management strategies. Similarly, although the software can account for climate change, the hybrid approach is only an approximation and has several shortcomings (see earlier). Other risks and uncertainties, such as natural disturbances and socio-economic developments, as well as features, such as genetic characteristics and species interactions in mixed and uneven-aged forests, cannot be taken into account. Nevertheless, if appropriate models become available, these factors could be included to a certain extent. For instance, a certain mixture of tree species and ages could be assigned to a pixel, as long as there is a model that can assign a corresponding development path, which restricts it to a predefined set of combinations (no real-time models), while in general, the spatial representation is restricted by not considering within-pixel variation. As a rule, the user should be mindful of the shortcomings and, because of the uncertainties and possible inaccuracies, interpret the results in relative terms. In addition, the uncertainties call for using Sim4Tree for adaptive management, whereby the quantitative optimization is complemented with expert knowledge and the planning is systematically adjusted to deviations and future developments.

Lastly, participatory elements occupy a central role in Sim4Tree. In the first place, the software has been designed to incorporate stakeholder opinions, which is materialized by the MCDA approach. This functionality is vital to the decision making process, although it should be acknowledged that Sim4Tree usually only forms a part of the participatory process and should be dynamically complemented with other methods and stakeholder interactions to realize a full-fledged decision making process. This was clearly illustrated by the Bosland case study. Furthermore, the fact that Sim4Tree does not try to cover the entire participatory process keeps it from being too specific and, as such, bears the advantage that it can be creatively used in various applications. Secondly, stakeholders' and potential users' feedback and suggestions for DSS contents and features have been of prime importance throughout the software development and have been decisive for the software design in all of its aspects. The attempt to satisfy differing user needs and to favor effective widespread use is above all reflected by the three defined decision levels, N1, N2 and N3. Much effort has been put on user-friendliness (e.g., intuitive stand-alone GUI, various visualization options, including GIS, extensive manual and documentation) and on maintaining transparency and credibility for both experts and non-experts through the straightforward methodology (e.g., initialization, yield tables and visualized results are all very intuitive; the MCDA approach leads to a transparent and consistent evaluation). As a result, Sim4Tree might essentially contribute to decision making, as long as its possibilities and limitations within the participatory process are well understood.

In summary, with Sim4Tree v2.5, we have a DSS at our disposal that can aid in the participatory decision making process on the strategic policy and management of forest regions and complexes in Flanders. Despite various shortcomings and uncertainties, its inherent structural flexibility implies great potential for further improvement of the performance and for extension of the applicability, which is all the more important given future prospects of a continued increase in the demand for integration and participation and in computer power. Related to this, its low requirement for input data, at least on decision levels N1 and N2, is an important asset in light of expected increasing labor costs for inventories 
and field observations. Future software adaptations will primarily focus on shortcomings that impede Sim4Tree's use on a larger scale. In fact, a list of possible future adaptations was compiled, and these were ranked according to their importance and feasibility. This revealed, amongst a total of 75 suggestions, four absolute priorities: incorporating models other than the yield tables, operationalizing decision level N3, accounting for sub-optimal tree densities (no maximal basal area) and implementing various cost-benefit profiles. In addition, future adaptation will be steered by feedback and suggestions from users.

\section{Conclusions}

This paper has presented the Sim4Tree DSS, which supports participatory decision making for forest management and policy. It provides simulations of forest development, ecosystem services potential and economic performance through time, at multiple spatio-temporal scales, under user-defined management and climate regimes, and allows for an optimization by ranking different scenarios. The current Sim4Tree v2.5 is parameterized for the region of Flanders, Belgium, and has achieved substantial merits towards including participatory approaches and integrating knowledge sources within a toolbox. Its lack of robustness remains the weak point, however. Nevertheless, the general plug-in architecture of Sim4Tree allows for further adaptions and, correspondingly, (even) further improvements with regard to these three aspects. Rather than focusing on ad hoc needs of particular projects, the software is designed to keep up with newly emerging decision problems and questions at various spatio-temporal scales.

\section{Acknowledgments}

We thank the Flemish Fund for Science and Technology (IWT) and all members of the IWT Strategic Basic Research (SBO) Sim4Tree consortium, including Gaby Deckmyn and Reinhart Ceulemans (University of Antwerp), Dries Vansteenkiste and Joris Van Acker (Ghent University), Marc Devrieze and Joost Malfliet (KU Leuven, University of Leuven), for their contributions to the first version of Sim4Tree. We also thank the Flemish Agency for Nature and Forests (Ondersteunend Centrum van het Agentschap voor Natuur en Bos, OC-ANB) for the financial support and Wim Aertsen, Pieter Moonen, Lieve Borremans and Thomas Van de Peer for their contributions to Sim4Tree v2.5. Lastly, we want to thank all members of the different stakeholder commissions, which delivered input for the development of Sim4Tree along the way.

\section{Author Contributions}

Floris Dalemans contributed to the climate functionalities of the software, to the user manual and other documentation, to the software tests and to the list of future possible adaptations and first-authored the paper. Paul Jacxsens designed and implemented the database in PostgreSQL RDBMS, designed and coded the software functionalities in the C\#.NET framework and contributed to the documentation, software tests and future possible adaptations. Pieter Moonen was a principal investigator in the Bosland case study and wrote parts of the corresponding section of the paper. Vincent Kint initiated Sim4Tree development and contributed together with Bart Muys to its first version; together with Jos Van 
Orshoven, they also coordinated the development of v2.5, contributed to the documentation and proofread and contributed to the drafts of the paper. Moreover, Vincent Kint contributed to the software tests and future possible adaptations and wrote parts of the Sim4Tree decision support system section of the paper.

\section{Conflicts of Interest}

The authors declare no conflict of interest.

\section{References}

1. Farrell, E.P.; Führer, E.; Ryan, D.; Andersson, F.; Hüttl, R.; Piussi, P. European forest ecosystems: Building the future on the legacy of the past. For. Ecol. Manag. 2000, 132, 5-20.

2. Cubbage, F.; Harou, P.; Sills, E. Policy instruments to enhance multi-functional forest management. For. Policy Econ. 2007, 9, 833-851.

3. Hahn, W.A.; Knoke, T. Sustainable development and sustainable forestry: Analogies, differences, and the role of flexibility. Eur. J. For. Res. 2010, 129, 787-801.

4. Department of Forest Resource Management, Swedish University of Agricultural Sciences. Computer-Based Tools for Supporting Forest Management; Borges, J.G., Nordström, E.M., Garcia Gonzalo, J., Hujala, T., Trasobares, A., Eds.; Department of Forest Resource Management, Swedish University of Agricultural Sciences: Umea, Sweden, 2014; p. 503.

5. De Groot, R.S.; Wilson, M.A.; Boumans, R.M. J. A typology for the classification, description and valuation of ecosystem functions, goods and services. Ecol. Econ. 2002, 41, 393-408.

6. Spiecker, H. Silvicultural management in maintaining biodiversity and resistance of forests in Europe-Temperate zone. J. Environ. Manag. 2003, 67, 55-65.

7. Bugmann, H.; Palahí, M.; Bontemps, J.-D.; Tomé, M. Trends in modeling to address forest management and environmental challenges in Europe: Introduction. For. Syst. 2010, 19, 3-7.

8. Pretzsch, H.; Grote, R.; Reineking, B.; Rötzer, T.H.; Seifert, S.T. Models for forest ecosystem management: A European perspective. Ann. Bot. 2008, 101, 1065-1087.

9. Fürst, C.; Lorz, C.; Vacik, H.; Potocic, N.; Makeschin, F. How to support forest management in a world of change: Results of some regional studies. Environ. Manag. 2010, 46, 941-952.

10. Reynolds, K.M.; Twery, M.; Lexer, M.J.; Vacik, H.; Ray, D.; Shao, G.; Borges, J.G. Decision support systems in forest management. In Handbook on Decision Support Systems 2; Burstein, F., Holsapple, C.W., Eds.; Springer: Berlin, Germany, 2008; pp. 499-533.

11. Muys, B.; Hynynen, J.; Palahí, M.; Lexer, M.J.; Fabrika, M.; Pretzsch, H.; Gillet, F.; Briceño, E.; Nabuurs, G.J.; Kint, V. Simulation tools for decision support to adaptive forest management in Europe. For. Syst. 2010, 19, 86-99.

12. Sheppard, S.R.J.; Achiam, C.M. Public participation in forest decision making. In Encyclopedia of Forest Sciences; Evans, J., Youngquist, J., Eds.; Academic Press/Elsevier: Oxford, UK, 2004; pp. 1173-1182.

13. Lawrence, A.; Stewart, A. Sustainable forestry decisions: On the interface between technology and participation. Math. Comput. For. Nat. Sci. 2011, 3, 42-52. 
14. Menzel, S.; Nordström, E.-M.; Buchecker, M.; Marques, A.; Saarikoski, H.; Kangas, A. Decision support systems in forest management: Requirements from a participatory planning perspective. Eur. J. For. Res. 2012, 131, 1367-1379.

15. Sheppard, S.R.J.; Meitner, M. Using multi-criteria analysis and visualisation for sustainable forest management planning with stakeholder groups. For. Ecol. Manag. 2005, 207, 171-187.

16. Marques, A.F.; Ficko, A.; Kangas, A.; Rosset, C.; Ferreti, F.; Rasinmaki, J.; Packalen, T.; Gordon, S. Empirical guidelines for forest management decision support systems based on the past experiences of the expert's community. For. Syst. 2013, 22, 320-339.

17. Vacik, H.; Lexer, M.J. Past, current and future drivers for the development of decision support systems in forest management. Scand. J. For. Res. 2014, 29, 2-19.

18. Packalen, T.; Marques, A.F.; Rasinmäki, J.; Rosset, C.; Mounir, F.; Rodriguez, L.C.E.; Nobre, S.R. A brief overview of forest management decision support systems (FMDSS) listed in the FORSYS wiki. For. Syst. 2013, 22, 263-269.

19. Segura, M.; Ray, D.; Maroto, C. Decision support systems for forest management: A comparative analysis and assessment. Comput. Electron. Agric. 2014, 101, 55-67.

20. Marques, A.F.; Rosset, C.; Rasinmaki, J.; Vacik, H.; Gordon, S.; Nobre, S.; Falcao, A.; Weber, D.; Granitzer, M.; Eriksson, L.O. Collaborative development of a semantic wiki on forest management decision support. Scand. J. For. Res. 2014, 29, 30-43.

21. Calama, R.; Tomé, M.; Sanchez-Gonzalez, M.; Miina, J.; Spanos, K.; Palahí, M. Modelling non-wood forest products in Europe : A review. For. Syst. 2010, 19, 69-85.

22. Mäkelä, A.; Grace, J.C.; Deckmyn, G.; Kantola, A.; Campioli, M. Simulating wood quality in forest management models. For. Syst. 2010, 19, 48-68.

23. Reynolds, K.M.; Schmoldt, D.L. Computer-aided decision making. In Computer Applications in Sustainable Forest Management; Shao, G., Reynolds, K.M., Eds.; Springer: Netherlands, 2006; pp. 143-169.

24. Rammer, W.; Schauflinger, C.; Vacik, H.; Palma, J.H.N.; Garcia-Gonzalo, J.; Borges, J.G.; Lexer, M.J. A web-based ToolBox approach to support adaptive forest management under climate change. Scand. J. For. Res. 2014, 29, 96-107.

25. Reynolds, K.M.; Borges, J.G.; Vacik, H.; Lexer, M.J. ICT in forest management and conservation. In Information Technology and the Forest Sector. IUFRO World Series Volume 18; Hetemaki, L., Nilsson, S., Eds.; International Union of Forest Research Organizations: Vienna, Austria, 2005; pp. $150-171$.

26. Bhargava, H.K.; Power, D.J.; Sun, D. Progress in web-based decision support technologies. Decis. Support Syst. 2007, 43, 1083-1095.

27. Lexer, M.J.; Seidl, R. Addressing biodiversity in a stakeholder-driven climate change vulnerability assessment of forest management. For. Ecol. Manag. 2009, 258, S158-S167.

28. Fontes, L.; Bontemps, J.-D.; Bugmann, H.; van Oijen, M.; Gracia, C.; Kramer, K.; Lindner, M.; Rötzer, T.; Skovsgaard, J.P. Models for supporting forest management in a changing environment. For. Syst. 2010, 19, 8-29.

29. Hanewinkel, M.; Peltola, H.; Soares, P.; Gonzalez-Olabarria, J.R. Recent approaches to model the risk of storm and fire to European forests and their integration into simulation and decision support tools. For. Syst. 2010, 19, 30-47. 
30. Kramer, K.; Buiteveld, J.; Forstreuter, M.; Geburek, T.; Leonardi, S.; Menozzi, P.; Povillon, F.; Schelhaas, M.J.; Teissier du Cros, E.; Vendramin, G.G.; et al. Bridging the gap between ecophysiological and genetic knowledge to assess the adaptive potential of European beech. Ecol. Modell. 2008, 216, 333-353.

31. Kramer, K.; van der Werf, D.C. Equilibrium and non-equilibrium concepts in forest genetic modelling: Population- and individually-based approaches. For. Syst. 2010, 19, 100-112.

32. Uran, O.; Janssen, R. Why are spatial decision support systems not used? Some experiences from the Netherlands. Comput. Environ. Urban Syst. 2003, 27, 511-526.

33. IPCC. Special Report on Emissions Scenarios; Nakicenovic, N., Swart, R., Eds.; Cambridge University Press: Cambridge, UK, 2000; p. 570.

34. Van Orshoven, J.; Gilliams, S.; Muys, B.; Stendahl, J.; Skov-Petersen, H.; van Deursen, W. Support of decisions on afforestation in north-western Europe with the Afforest-sDSS. In Environmental Effects of Afforestation in North-Western Europe; Heil, G.W., Muys, B., Hansen, K., Eds.; Springer: Dordrecht, The Netherlands, 2007; pp. 227-247.

35. Jansen, J.J.; Sevenster, J.; Faber, P.J. Opbrengsttabellen Voor Belangrijke Boomsoorten in Nederland; IBN-DLO: Wageningen, The Netherlands, 1996; p. 240.

36. Meuleman, B.; Dufrane, F.; Carletti, G. Productietabel Voor Corsicaanse den in Vlaanderen; LISEC: Genk, Belgium, 1991; p. 91.

37. Malczewski, J. GIS and Multicriteria Decision Analysis; Wiley: New York, NY, USA, 1999; p. 408.

38. Mendoza, G.A.; Martins, H. Multi-criteria decision analysis in natural resource management: A critical review of methods and new modelling paradigms. For. Ecol. Manag. 2006, 230, 1-22.

39. De Meyer, A.; Estrella, R.; Jacxsens, P.; Deckers, J.; Van Rompaey, A.; Van Orshoven, J. A conceptual framework and its software implementation to generate spatial decision support systems for land use planning. Land Use Policy 2013, 35, 271-282.

40. Estrella, R.; Cattrysse, D.; van Orshoven, J. Comparison of three ideal point-based multi-criteria decision methods for afforestation planning. Forests 2014, 5, 3222-3240.

41. Mustajoki, J.; Hämäläinen, R.P. Web-HIPRE: Global decision support by value tree and AHP analysis. INFOR J. 2000, 38, 208-220.

42. Tervonen, T. JSMAA: Open source software for SMAA computations. Int. J. Syst. Sci. 2014, 45, 69-81.

43. Gilliams, S.; Raymaekers, D.; Muys, B.; van Orshoven, J. Comparing multiple criteria decision methods to extend a geographical information system on afforestation. Comput. Electron. Agric. 2005, 49, 142-158.

(C) 2015 by the authors; licensee MDPI, Basel, Switzerland. This article is an open access article distributed under the terms and conditions of the Creative Commons Attribution license (http://creativecommons.org/licenses/by/4.0/). 